\title{
Engineering broad-spectrum digestion of polyuronides from an exolytic polysaccharide lyase
}

\author{
Logan C. MacDonald', Elizabeth B. Weiler ${ }^{1}$ and Bryan W. Berger ${ }^{1,2^{*}}$
}

\begin{abstract}
Background: Macroalgae represents a promising source of fermentable carbohydrates for use in the production of energy efficient biofuel. The primary carbohydrate in brown algae is the uronic acid-containing alginate, whereas green algae contains a significant amount of glucuronan. A necessary step in the conversion of these polyuronides to bioethanol is saccharification, which can be achieved by enzymatic or chemical degradation.
\end{abstract}

Results: Polysaccharide lyases are a class of enzymes which cleave uronic acid-containing glycans via a $\beta$-elimination mechanism, acting both endo- and exolytically on their substrates. In the present work, we characterize a putative alginate lyase from Stenotrophomonas maltophilia K279a (Smlt2602) and describe a H208F mutant that, in addition to cleaving alginate-based substrates, displays significant, exolytic glucuronan activity.

Conclusions: To our knowledge this is the first polysaccharide lyase to act exolytically on glucuronan and is an attractive candidate for the broad-spectrum digestion of polyuronides into fermentable monomers.

Keywords: Alginate, Polysaccharide, Alginate lyase, Uronic acid, Stenotrophomonas maltophilia

\section{Background}

Rising energy costs, diminishing fossil fuel resources, and increased greenhouse gas emissions have magnified the need to produce environmentally friendly biofuels via the microbial fermentation of readily available biomass. Macroalgae is a promising biomass source due to its cultivation requiring no arable land or fresh water, minimizing any economical cost due to loss of farmland currently dedicated to food production [1]. Algae is also capable of sequestering significant amounts of carbon from the atmosphere and metabolizing nutrients found in industrial effluents and municipal wastewater [2]. Furthermore, the lack of lignin in algae allows for the simple and inexpensive extraction of polysaccharides [3]. The primary carbohydrate in brown algae is alginate, whereas green algae contains significant amounts of glucuronan

\footnotetext{
*Correspondence: bwb209@lehigh.edu

${ }^{2}$ Department of Chemical and Biomolecular Engineering, Lehigh University, B320 lacocca Hall, 111 Research Drive, Bethlehem, PA 18015, USA

Full list of author information is available at the end of the article
}

and its derivatives [3, 4]. Alginate is a linear copolymer comprised repeating units of $\mathrm{D}$-mannuronic acid (ManA) and L-guluronic acid (GulA) arranged in three block types: repeating units of poly- $\beta$-D-ManA, repeating units of poly- $\alpha-\mathrm{L}-\mathrm{GulA}$, and alternating units of ManA and GulA (poly-MG). Glucuronan is a homopolymer of D-glucuronic acid (GlcA), the C2 epimer of ManA [5]. Saccharification of these uronic acid-containing polysaccharides is a necessary step in their conservation to bioethanol [6].

Carbohydrate modifying enzymes offer a promising approach to produce fermentable monomers [7]. Polysaccharide lyases (PLs) are a class of carbohydrate modifying enzymes which cleave uronic acid-containing polysaccharides via a $\beta$-elimination mechanism, resulting in the formation of a double between $\mathrm{C} 4$ and $\mathrm{C} 5$ of the sugar group at the new nonreducing end [8]. This unsaturated product can be detected by monitoring absorbance at $235 \mathrm{~nm}$ [9]. Successful high-yield recombinant expression and purification, ease of product detection, and selectively for uronic acid monomers, which 
are found in many difficult to ferment glycans, make this class of enzymes advantageous for polysaccharide processing $[5,10,11]$. Previously, we reported an endolytic polysaccharide lyase (Smlt1473) from Stenotrophomonas maltophilia K279a that exhibited a unique $\mathrm{pH}$-sensitive substrate specificity that could be significantly modified through the mutation of residues located in the active site cleft but not directly involved in the catalytic process. We demonstrated that single-point mutations H221F and R312L resulted in increased activity and specificity toward poly-ManA and poly-GlcA, respectively. These and other residues were selected based on sequence alignment and homology modeling and were hypothesized to participate in substrate binding and the precise positioning of the cleavable glycosidic bond with respect to the catalytic tetrad $[5,12]$.

In the present work, we characterize an exolytic polysaccharide lyase (Smlt2602) from S. maltophilia K279a and undertake a similar approach. Wild-type Smlt2602 (WT) acts on alginate and each of its block structures, with highest activity toward poly-ManA. Mutation of putative catalytic residues reveals that $\mathrm{Tyr}^{264}$ and $\mathrm{His}^{418}$ likely play the role of the general acid and base, respectively. Interestingly, we isolated a $\mathrm{H} 208 \mathrm{~F}$ mutant that exhibits significant exolytic activity toward poly-GlcA, a non-alginate-based substrate that Smlt2602 is virtually inactive against. Thus, unlike Smlt1473 in which substrate specificity was only modified, we are able to engineer significant novel activity into Smlt2602 via a sequence and homology model-based approach. To our knowledge, this is the first characterized bacterial lyase to demonstrate exolytic alginate and poly-GlcA activity and is another example of the structural plasticity in certain polysaccharide lyases which could be applied to the design of highly active and specific enzymes for use in glycan saccharification.

\section{Methods \\ Homology modeling}

The exolytic alginate lyase Alg17c (Protein Data Bank 4OJZ) from Saccharophagus degradans 2-40 was chosen as a template for constructing a homology model of Smlt2602 due to having the highest sequence identity $(50 \%)$ among polysaccharide lyases with solved crystal structures [13]. The model was built in SWISS-MODEL [14-16], and all images were generated in PyMOL [17].

\section{Subcloning, expression, and purification}

Unless otherwise stated, standard molecular biology techniques were used for subcloning and site-directed mutagenesis [18]. An E. coli codon-optimized nucleotide sequence of smlt2602 (NCBI GeneID 6,393,623) was subcloned into pET28a(+) (Invitrogen) as a BamHI-XhoI insert. Mutagenic primers were designed via PrimerX (bioinformatics.org/primer), and point mutations were generated via QuikChange II Site-directed Mutagenesis kit (Agilent Technologies). Mutations were confirmed by DNA sequencing (GeneWiz). Expression and purification were carried out in E. coli BL21(DE3) cells as described previously [5]. Fractions were assayed for protein content via Bradford reagent (Bio-Rad), SDS-PAGE, and immunoblotting as described previously [5]. Samples containing purified Smlt2602 were pooled together and dialyzed against $4 \mathrm{~L}$ of $20 \mathrm{mM}$ sodium phosphate buffer ( $\mathrm{pH} 8.5)$, $100 \mathrm{mM} \mathrm{NaCl}, 5 \% v / v$ glycerol, $20 \mathrm{mM}$ imidazole for $20 \mathrm{~h}$ at $4{ }^{\circ} \mathrm{C}$, and then $20 \mathrm{mM}$ sodium phosphate buffer (pH 8.5) for $40 \mathrm{~h}$ at $4{ }^{\circ} \mathrm{C}$ with one buffer exchange. Protein concentration was determined via absorbance measurements at $280 \mathrm{~nm}$ and molar extinction coefficients estimated from primary amino acid sequences [19].

\section{Polysaccharide substrates}

Low viscosity sodium alginate and heparin were obtained from Sigma-Aldrich. Chondroitin sulfate and poly-GalA were obtained from Alfa Aesar. Hyaluronic acid, potassium salt from human umbilical cord, and heparin sulfate were obtained from MP biomedicals and sagent pharmaceuticals, respectively. Poly-GlcA was prepared from Avicel PH-105 NF (FMC Biopolymer) and its structure confirmed by ${ }^{1} \mathrm{H}$ NMR as described previously [5]. PolyManA, poly-GulA, and poly-MG were prepared via partial acid hydrolysis of sodium alginate, and structure was confirmed by ${ }^{1} \mathrm{H}$ NMR as described previously [5]. All prepared polysaccharide samples were dialyzed against $4 \mathrm{~L}$ of $\mathrm{dd}_{2} \mathrm{O}$ for $40 \mathrm{~h}$ at $4{ }^{\circ} \mathrm{C}$ with one buffer exchange, lyophilized, and stored as powders at $4{ }^{\circ} \mathrm{C}$ until needed.

\section{Enzyme activity assays}

The $\beta$-elimination mechanism of polysaccharide lyases creates a double bond between $\mathrm{C} 4$ and $\mathrm{C} 5$ of the sugar ring at the newly formed nonreducing end whose accumulation can be monitored by measuring the change in absorbance at $235 \mathrm{~nm}$ [9]. Absorbance measurements were taken in $1 \mathrm{~s}$ intervals over the course of at least $5 \mathrm{~min}$ via an Ultrospec $3300 \mathrm{UV}-\mathrm{Vis}$ spectrophotometer with a detection limit of 0.001 absorbance units at $235 \mathrm{~nm}$ per minute, as described previously [5]. One unit of enzyme activity was defined as an increase in absorbance at $235 \mathrm{~nm}$ of 1.0 per minute at $25^{\circ} \mathrm{C}(1$ unit $=1$ $\left.\Delta \mathrm{A}_{235 \mathrm{~nm} \mathrm{~min}}{ }^{-1}\right)[5,10]$. In general, $3.5 \mu \mathrm{g}$ of purified wild-type and mutant Smlt2602 was added to a solution containing $1 \mathrm{mg} / \mathrm{mL}$ substrate in a final volume of $350 \mu \mathrm{L}$. The $\mathrm{pH}$ of all reactions was controlled via specific buffers for a given $\mathrm{pH}$ range at a total ionic strength of $20 \mathrm{mM}$ (acetate for $\mathrm{pH} 4-6$, phosphate for $\mathrm{pH} 6-8.5$, Tris for $\mathrm{pH} 7-9$, and glycine for $\mathrm{pH} 9-10)$. Additionally, 
the Michaelis constant $\left(K_{M}\right)$ and turnover number $\left(k_{\mathrm{CAT}}\right)$ of wild-type Smlt2602 and H208F mutant against alginate, poly-ManA, poly-GulA, poly-MG, and poly-GlcA were determined by varying substrate concentration. An extinction coefficient of $6150 \mathrm{M}^{-1} \mathrm{~cm}^{-1}$ at $235 \mathrm{~nm}$ was used to convert absorbance values to product concentration $[11,12]$. Initial rates $\left(v_{i}\right)$ were fit to the Michaelis-Menten equation, $v=k_{\mathrm{CAT}} E_{0} S /\left(K_{M}+S\right)$ with a generalized reduced gradient (GRG2) nonlinear optimization program [20]. The $R^{2}$ and correlation values for each enzymes against each substrate were greater than 0.989 and 0.993 , respectively. All reactions were carried out in triplicate and error is reported as standard deviation. Lyase activity was independently determined via the thiobarbituric acid (TBA) method as described previously $[12,21]$.

\section{Circular dichroism}

Enzyme samples $(300 \mu \mathrm{L})$ at $250 \mu \mathrm{g} / \mathrm{mL}$ in $20 \mathrm{mM}$ sodium phosphate buffer ( $\mathrm{pH} 8.5$ ) were added to $1-\mathrm{mm}$ path length quart cuvettes (Starna), and ellipticity was measured from 190 to $260 \mathrm{~nm}$ in a J-815 circular dichroism spectrometer (JASCO) at a scan speed of $100 \mathrm{~nm} /$ min with three accumulations per sample.

\section{High-performance liquid chromatography (HPLC) of unsaturated sugar products}

Size-exclusion chromatography experiments were performed on an Agilent 1100 series HPLC value system equipped with a 96-well autosampler and UV-visible detector. Wild-type and mutant Smlt2602 at a final concentration of $10 \mu \mathrm{g} / \mathrm{mL}$ was added to poly-ManA or poly-GlcA at a final concentration of $1 \mathrm{mg} / \mathrm{mL}$ in $20 \mathrm{mM}$ sodium phosphate buffer, $\mathrm{pH} 8.5$, and incubated for $6 \mathrm{~h}$ at room temperature. The reaction mixture $(15 \mu \mathrm{L})$ was injected into a TSKgel SuperOligoPW column (Tosoh) equipped with corresponding guard column. The mobile phase was $20 \mathrm{mM}$ sodium phosphate buffer $(\mathrm{pH}$ 8.5) plus $250 \mathrm{mM} \mathrm{NaCl}$ and the flow rate was $0.3 \mathrm{~mL} / \mathrm{min}$. Unsaturated uronic acid products were detected by absorbance at $235 \mathrm{~nm}$. Polyethylene glycol (molecular weight 600, 1000 and 2000; Alfa Aesar), D-glucuronic acid (Acros Organics) and xylose (Spectrum) were used as molecular weight standards to generate a calibration curve.

\section{Results and discussion}

Substrate Specificity Analysis Revealed that Smlt2602 is an Alginate-Specific Lyase

An $E$. coli codon-optimized nucleotide sequence of smlt2602 was subcloned into pET28a(+) as a BamHIXhoI insert without a stop codon, resulting in the recombinant lyase containing a $\mathrm{C}$-terminal $\mathrm{His}_{6}$ tag. Expression was carried out in E. coli BL21(DE3) cells at $18{ }^{\circ} \mathrm{C}$, and
Smlt2602-His ${ }_{6}$ was purified by passing soluble cell lysate over a $\mathrm{Ni}^{2+}$-bound chelating Sepharose column as described previously [5]. Approximately $60 \mathrm{mg}$ of enzyme was purified per liter of induced cultured. An SDS-PAGE and anti-His ${ }_{6}$ immunoblot (Fig. 1a) confirmed the presence of purified Smlt2602 at the expected molecular weight of $80.8 \mathrm{kDa}$, predicted from the primary amino acid sequence of Smlt2602--His 6 without signaling peptide [19].

The enzymatic activity of purified Smlt2602 was tested against the following ten polyuronides: alginate, poly-ManA, poly-GulA, poly-MG, poly-GlcA, poly- $\alpha-$ D-galacturonic acid (poly-GalA), hyaluronan, heparin, heparan sulfate, and chondroitin sulfate. Of these substrates, significant activity was measured for alginate and each of its block structures (Fig. 1b; Table 1) at an optimal pH of 8.5 (see Additional file 1: Figure S1 for relative activity against poly-ManA as a function of $\mathrm{pH}$ ). The highest overall specific activity $(661.9 \pm 29.1$ units/mg) was for poly-ManA, which is among the highest reported for exolytic poly-ManA degradation [22-24]. Smlt2602 exhibited approximately half the activity toward alginate $(328.3 \pm 27.0$ units $/ \mathrm{mg})$ and poly-MG $(289.3 \pm 9.8$ units $/ \mathrm{mg}$ ) and the lowest activity toward poly-GulA (190.3 \pm 8.3 units/mg). No product formation was detected for the remaining substrates with the exception of poly-GlcUA, toward which Smlt2602 displayed minimal yet measurable activity $(2.8 \pm 0.4$ units $/ \mathrm{mg}$, less than $0.5 \%$ that of polyManA) (Fig. 1b; Table 1 ).

\section{Identification of putative catalytic and substrate-binding residues via homology modeling}

Polysaccharide lyases are divided into 22 families based on sequence, with the structure of at least one protein in each family determined. Comparison of solved crystal structures

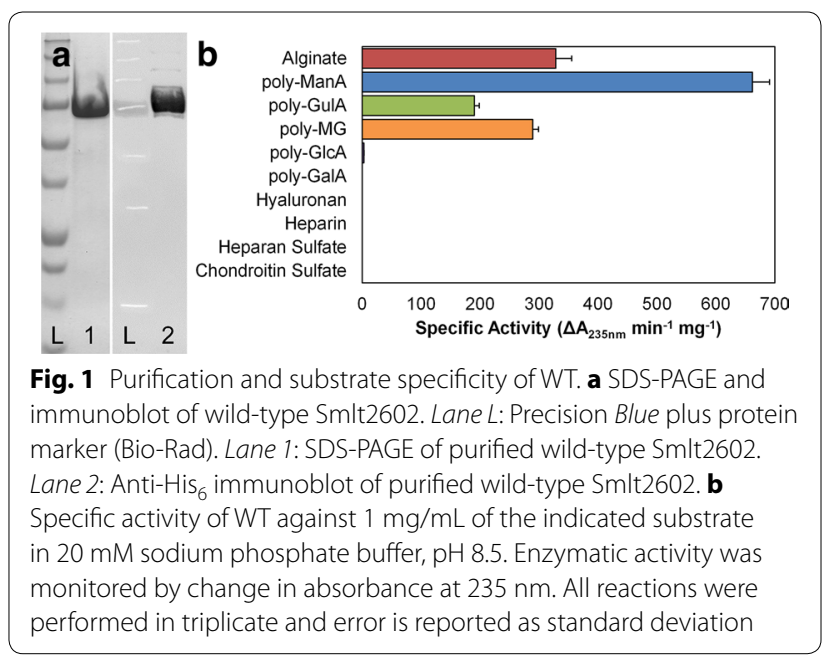


Table 1 Kinetic parameters of WT and H208F mutant

\begin{tabular}{|c|c|c|c|c|c|c|}
\hline & \multicolumn{3}{|l|}{ WT } & \multicolumn{3}{|l|}{ H208F } \\
\hline & $k_{\mathrm{CAT}}\left(\mathrm{s}^{-1}\right)$ & $K_{M}(\mathrm{mM})$ & $k_{\mathrm{CAT}} / K_{M}$ & $k_{\mathrm{CAT}}\left(\mathrm{s}^{-1}\right)$ & $K_{M}(\mathrm{mM})$ & $k_{\mathrm{CAT}} / K_{M}$ \\
\hline Alginate & 34.8 & 0.67 & 52.2 & 14.6 & 1.35 & 10.8 \\
\hline PolyManA & 62.2 & 0.41 & 151.8 & 21.3 & 0.41 & 51.5 \\
\hline Poly-GulA & 34.6 & 5.99 & 5.8 & 1.4 & 2.22 & 0.7 \\
\hline Poly-MG & 22.2 & 0.57 & 38.7 & 2.0 & 0.98 & 2.1 \\
\hline Poly-GlcA & 0.4 & 1.40 & 0.3 & 6.1 & 0.57 & 10.6 \\
\hline
\end{tabular}

WT and H208F mutant were added to 10 different solutions containing $7.8-4000 \mu \mathrm{g} / \mathrm{mL}$ substrate in $20 \mathrm{mM}$ sodium phosphate buffer at pH 8.5 for alginate-based polysaccharides, and $\mathrm{pH} 8$ for poly-GlcA. Enzymatic activity was monitored by absorbance at $235 \mathrm{~nm}$, and all reactions were performed in triplicate. Turnover rate $\left(k_{\text {CAT }}\right)$ was calculated using an extinction coefficient of $6150 \mathrm{M}^{-1} \mathrm{~cm}^{-1}$ for the unsaturated product $[11,12] . K_{M}$ values are based upon an effective molecular weight of 176 for the monosaccharide unit, the smallest product formed by Smlt2602 during catalysis [12, 52]

reveals that lyases from different families contain vastly different secondary structure content and thus overall fold [8]. Smlt2602 is assigned to the PL-17 family, along with Alg17c from Saccharophagus degradans 2-40, whose structure has recently been solved and revealed to contain two domains: an $\mathrm{N}$-terminal imperfect $\alpha$-barrel and $\mathrm{C}$-terminal $\beta$-sheet domain $[13,25]$. The circular dichroism spectrum of purified Smlt2602-His ${ }_{6}$ is indicative of a protein containing both $\alpha$-helices and $\beta$-sheets (see Additional file 1: Figure S2 for CD spectra comparing wild-type and mutant Smlt2602). Prediction of the secondary structure from the measured spectrum estimated that Smlt2602- $\mathrm{His}_{6}$ is $30 \%$ $\alpha$-helical and $25 \% \beta$-sheet [26], in close agreement with the secondary structure content of Alg17c (39 \% $\alpha$-helical and $26 \% \beta$-sheet) [13]. Furthermore, Smlt2602 shares $50 \%$ sequence identity with Alg17c (Fig. 2, top). Given that Smlt2602 contains the expected secondary structure content for a polysaccharide lyase belonging to the PL-17 family and exhibits significant sequence homology with Alg17c, another member of the same family, the high-resolution crystal structure of Alg17c (Protein Data Bank 4OJZ) was selected as the template to construct a homology model of Smlt2602 (Fig. 2, bottom).

In spite of diverse substrate specificity, secondary structure content, and tertiary folds, the $\beta$-elimination mechanism of PLs is remarkably conserved across the 22 families. The reaction proceeds via three chemical steps: (i) neutralization of the negatively charged C5 carboxylate group on the +1 sugar ring, reducing the $\mathrm{p} K_{a}$ of $\mathrm{H} 5$, thereby facilitating its abstraction; (ii) removal of $\mathrm{H} 5$ by a general base; and (iii) electron transfer resulting in the formation of a double bond between $\mathrm{C} 4$ and $\mathrm{C} 5$ of the +1 sugar ring and simultaneous protonation of $\mathrm{O} 4$ by a general acid resulting in the cleavage of the $\mathrm{C4}-\mathrm{O}-\mathrm{C} 1$ glycosidic bond [27]. Biochemical and structural characterization of Alg17c identified a tyrosine residue ( $\mathrm{Tyr}^{258}$ ) that is predicted to act as the general acid. This assertion is supported by the fact that the residue is in the correct orientation with respect to the glycosdic bond to donate a proton to $\mathrm{O} 4$ during the catalytic process and mutation of this tyrosine to phenylalanine abolished enzymatic activity [13]. The corresponding tyrosine in Smlt2602 $\left(\mathrm{Tyr}^{264}\right)$ is highlighted in blue in Fig. 2 and was utilized as an anchor point to approximate the location of the tunnel-like active site in Smlt2602. Park et al. proposed that $\mathrm{Asn}^{201}$ and $\mathrm{His}^{202}$ act as the neutralizing groups, whereas $\mathrm{Tyr}^{450}$ acts as the general base. All three of these residues were conserved in Smlt2602 $\left(\mathrm{Asn}^{207}, \mathrm{His}^{208}\right.$, and $\mathrm{Tyr}^{455}$ ) and are highlighted in orange in Fig. 2.

Previous studies of PLs have revealed that polar and charged residues located in the active site cleft form hydrogen bonds and salt bridges with the hydroxyl and carboxyl groups of the substrate [8]. In addition, aromatic side chains undergo $\mathrm{C}-\mathrm{H} / \pi$ interactions with the sugar rings of the glycan substrate [28]. The summation of these interactions results in the precise positioning of the polysaccharide substrate with respect to the catalytically active residues for optimal enzymatic cleavage [29, 30]. Our own study of Smlt1473 confirmed that residues such as tyrosine, histidine, and arginine located in the active site cleft significantly influence substrate specificity and overall activity [12]. To this end, we identified eight putative substrate-binding residues $\left(\mathrm{Arg}^{143}, \mathrm{Gln}^{153}\right.$, $\mathrm{Leu}^{155}$, $\mathrm{Asn}^{201}, \mathrm{His}^{206}, \mathrm{Tyr}^{258}$, Tyr ${ }^{263}$, and $\mathrm{His}^{418}$ ) highlighted in orange in Fig. 2, whose side chains are within $10 \AA$ of the putative catalytic $\mathrm{Tyr}^{264}$ in the Smlt2602 homology model. Thus, a total of 12 residues were selected for analysis via site-directed mutagenesis in an effort to elucidate their role in Smlt2602, four of which are predicted to participate directly in the $\beta$-elimination mechanism $\left(\mathrm{Asn}^{207}\right.$, $\mathrm{His}^{208}$, $\mathrm{Tyr}^{264}$, and $\mathrm{Tyr}^{455}$ ) and eight of which are predicted to bind and properly position the substrate in the active site $\left(\mathrm{Arg}^{143}, \mathrm{Gln}^{153}, \mathrm{Leu}^{155}, \mathrm{Asn}^{201}, \mathrm{His}^{206}, \mathrm{Tyr}^{258}\right.$, $\mathrm{Tyr}^{263}$, and His ${ }^{418}$ ) (Fig. 2). 


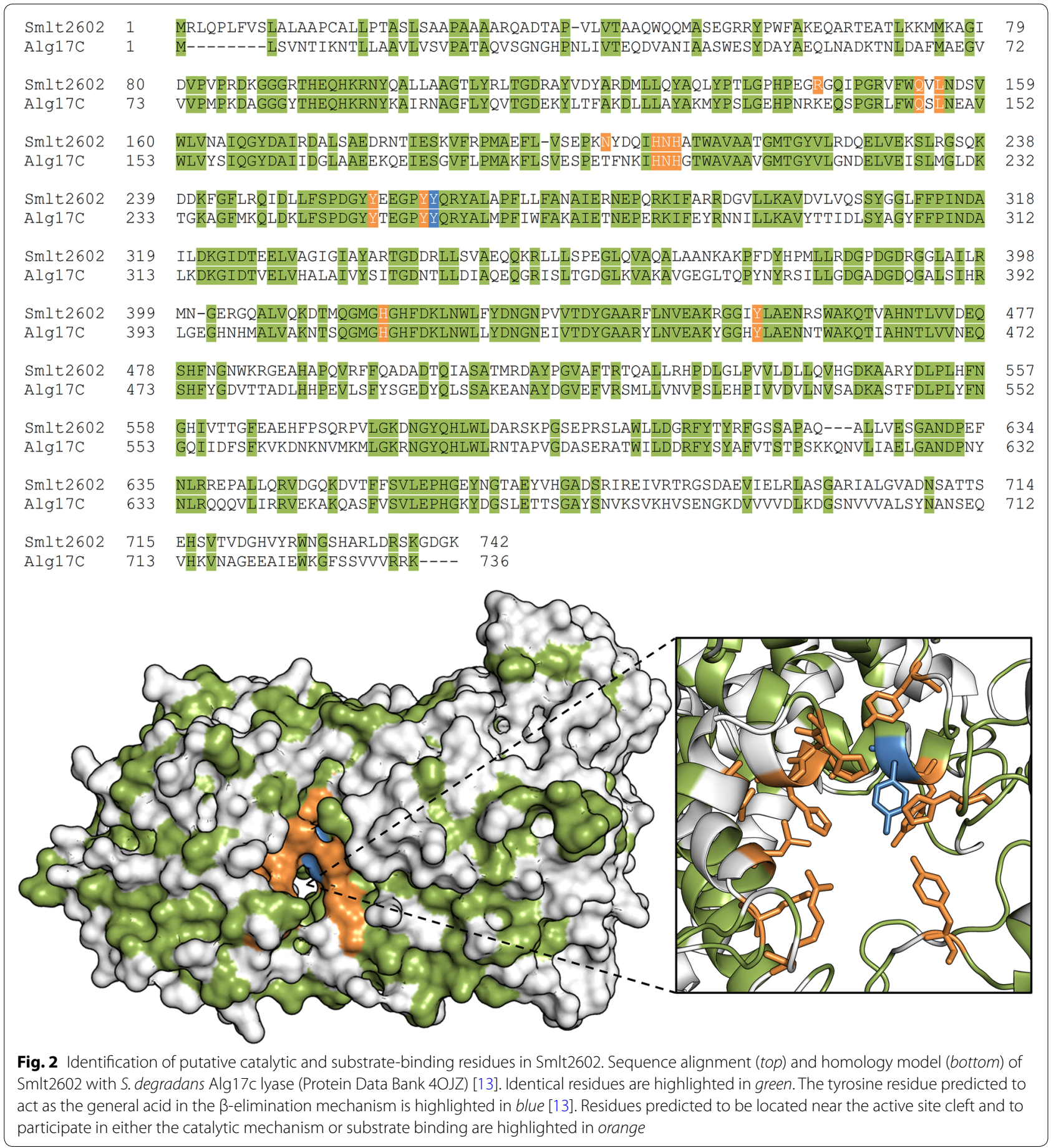

\section{Site-directed mutagenesis of putative catalytic residues indicates a tyrosine and histidine act as acid and base} As stated previously, the $\beta$-elimination mechanism of PLs involves three steps: neutralization of the negatively charged substrate, proton abstraction by a general base, and proton donation by a general acid [27]. Despite the significant structural diversity found among PLs, the residues responsible for each of the aforementioned steps appear to be highly conserved and fall into two general categories: (i) lyases that require a divalent cation (usually $\mathrm{Ca}^{2+}$ ) to act as the neutralizing group and (ii) lyases that do not require a divalent cation and instead one or more polar or charged residues (usually arginine, asparagine, or glutamine) in close proximity to the $\mathrm{C} 5$ 
carboxylate group act as the neutralizing group. In the former, a lysine or arginine acts as the base and water as the acid. In the latter, a tyrosine or histidine acts as the base and tyrosine as the acid [8]. For example, the crystal structure of Streptococcus pneumoniae hyaluronate lyase in complex with substrate revealed that the amide group of $\mathrm{Asn}^{239}$ fulfills the role of neutralization by interacting with the glycan carboxylate group in a bidentate fashion, and $\mathrm{Tyr}^{408}$ and $\mathrm{His}^{399}$ act as the acid and base, respectively [31]. Similarly, the crystal structure of Sphingomonas sp. A1-III alginate lyase in complex with its substrate revealed that in addition to $\mathrm{Asn}^{191}$, the guanidinium group of $\mathrm{Arg}^{239}$ acts as the neutralizing group, with a single tyrosine residue $\left(\mathrm{Tyr}^{246}\right)$ acting as both acid and base [32]. As discussed above, Park et al. proposed a third mechanism for Alg17c in which $\mathrm{Asn}^{201}$ and $\mathrm{His}^{202}$ act as neutralizing groups, with $\mathrm{Tyr}^{258}$ acting as the acid and $\mathrm{Tyr}^{450}$ as the base. Given that Smlt2602 and Alg17c both belong to PL-17, we hypothesize that Smlt2602 has a catalytic mechanism most similar to Alg17c, in which two tyrosine residues, $\mathrm{Ty}^{264}$ and $\mathrm{Tyr}^{455}$ in Smlt2602, play the role of general acid and base, respectively, and $\mathrm{Asn}^{207}$ and $\mathrm{His}^{208}$ neutralize the substrate (Fig. 2). To test this hypothesis, we prepared four inactivating mutants (N207L, H208F, Y264F, and Y455F) and measured their enzymatic activity against alginate and each of its block structures (Fig. 3a). In order to confirm that any observed variations in activity are not due to an overall distortion of the active site architecture, the $\mathrm{CD}$ spectrum of each mutant lyase (N207L, H208F, Y264F, H419F, and Y455F) was collected and compared to WT (see Additional file 1: Figure $S 2$ for CD spectra comparing wild-type and mutant Smlt2602). There was a less than $6 \%$ variation in the CD signal for each lyase, indicating that the overall secondary structure of Smlt2602 was not significantly perturbed by the point mutations.

The N207L and Y264F mutants are found to be completely inactive against alginate and all three block types (Fig. 3a), whereas the H208F mutant exhibits reduced activity toward each substrate tested, with approximately $30 \%$ WT activity against alginate and poly-ManA, and 5 and $8 \%$ WT activity against poly-GulA and poly-MG, respectively (Fig. 3a; Table 1 ). Our data are in close agreement with the kinetic parameters determined for mutants of the corresponding residues in Alg17c [13] and support the hypothesis that $\mathrm{Asn}^{207}$ and $\mathrm{His}^{208}$ act as neutralizing groups and $\mathrm{Tyr}^{264}$ as the proton donor during the catalytic process. Given that N207L is a null mutant while H208F retains significant enzymatic activity, the $\mathrm{Asn}^{207}$ residue likely plays a more crucial role in the neutralization of the glycan carboxylate. Moreover, mutation of $\mathrm{His}^{208}$ to Phe appears to have a significantly greater detrimental effect on poly-GulA and poly-MG activity compared to alginate and poly-ManA (Fig. 3a; Table 1), implying this residue is more critical for the neutralization of the +1 sugar carboxylate group when that sugar is GulA.

The Y455F mutant exhibits significantly diminished yet measurable activity toward alginate and each of its block types (Fig. 3a). More specificity, the mutant displays less

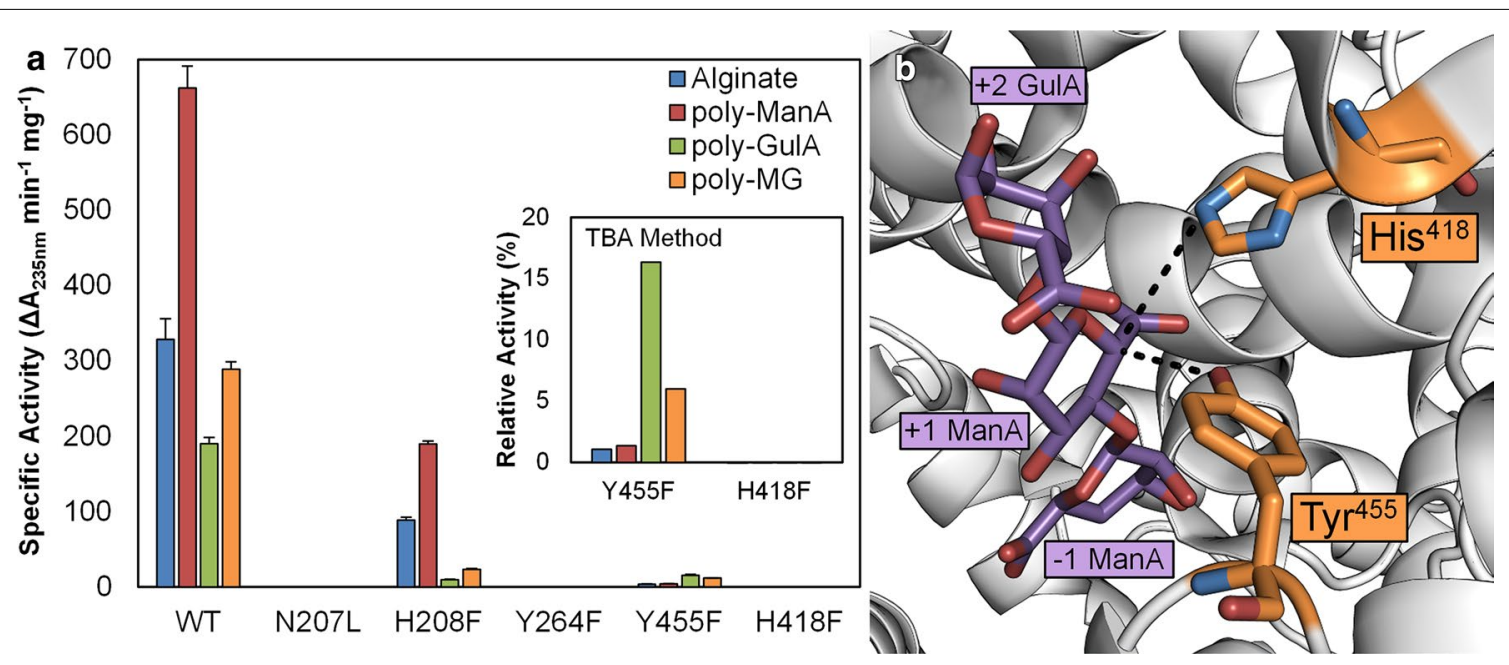

Fig. 3 Mutagenesis of putative catalytic residues. a Specific activity of wild-type Smlt2602 and N207L, H208F, Y264F, H418F, and Y455F mutants against $1 \mathrm{mg} / \mathrm{mL}$ alginate-based substrates in $20 \mathrm{mM}$ sodium phosphate buffer, pH 8.5. Enzyme activity was monitored by absorbance at $235 \mathrm{~nm}$ and confirmed via the TBA method (inset). For the TBA method, activity of WT against each substrate was taken to be $100 \%$. $\mathbf{b}$ Location of His ${ }^{418}$ and $\mathrm{Tyr}^{455}$ with respect to polysaccharide substrate, indicating each residue lies axial to the presumed location of the C5 proton (dashed black lines). Image generated from crystal structure of Alg17c from S. degradans 2-40 (Protein Data Bank 4OJZ) in complex with alginate trisaccharide (shown in purple). Residues in orange are His ${ }^{418}$ and Tyr ${ }^{450}$ of Alg17c which correspond to His ${ }^{418}$ and Tyr ${ }^{455}$ of Smlt2602 [13] 
than $1 \%$ alginate and poly-ManA activity compared to WT, yet approximately 8 and $4 \%$ WT activity toward poly-GulA and poly-MG, with specific activities of $15.3 \pm 1.4$ and $11.5 \pm 0.1$ units/mg, respectively (Fig. 3a; Table 1). Y445F activity was confirmed via the TBA method (Fig. 3a, inset). Although the measured enzymatic rates are minimal, the fact that the Y455F mutant demonstrates any residual activity suggests that $\mathrm{Tyr}^{455}$ may not act as the proton acceptor in the $\beta$-elimination mechanism of Smlt2602. To that end, we consulted the Smlt2602 homology model (Fig. 2) and the Alg17c crystal structure [13] in search of an additional residue that could possibly act as the base. We located a nearby histidine in Smlt2602 ( $\mathrm{His}^{418}$ ) whose corresponding histidine in $\mathrm{Alg} 17 \mathrm{c}\left(\mathrm{His}^{415}\right)$ lies on the same side of the substrate and is within $4.3 \AA$ of the C5 position of the +1 sugar ring, implying it could abstract the $\mathrm{C} 5$ proton during catalysis (Fig. 3b, dashed line). Therefore, we prepared a H418F mutant and tested its ability to cleave alginatebased substrates. We detect no increase in absorbance at $235 \mathrm{~nm}$ nor product formation via the TBA method, supporting the hypothesis that $\mathrm{His}^{418}$ is essential for catalysis (Fig. 3a).

Given the above results, we propose that $\mathrm{Tyr}^{264}$ acts as the proton donor in Smlt2602 and one of two scenarios is taking place with regards to the general base: (1) His ${ }^{418}$ acts as the proton acceptor, resulting in a catalytic mechanism similar to the hyaluronate lyase of Streptococcus pneumoniae [31]. In this regime, $\mathrm{Tyr}^{455}$ may stabilize an intermediate product of catalysis, explaining the diminished yet measurable activity of the Y455F mutant; (2) $\mathrm{Tyr}^{455}$ acts as the proton donor, resulting in a catalytic mechanism similar to Alg17c [13]. In this regime, the $\mathrm{His}^{418}$ residue may act as a surrogate, yet less efficient, proton acceptor in the absence of $\mathrm{Tyr}^{264}$, also explaining the diminished yet measureable activity of the Y445F mutant. In an effort to elucidate which mechanism is more likely, we considered the fact that the ionizable residue acting as the general base must be deprotonated at the $\mathrm{pH}$ at which the enzymatic reaction occurs. Therefore, we estimated the $\mathrm{p} K_{a}$ of the phenolic side of $\mathrm{Tyr}^{455}$ and the imidazole ring of $\mathrm{His}^{418}$ using PROPKA [33-36], which predicts shifts in $\mathrm{p} K_{a}$ resulting from interactions with the microenvironment surrounding the residue of interest [37]. Based on the Smlt2602 homology model (Fig. 2), the $\mathrm{p} K_{a}$ of $\mathrm{Tyr}^{455}$ was predicted to be shifted to 17.6, whereas the $\mathrm{p} K_{a}$ of $\mathrm{His}^{418}$ was 5.1. Similarly, the model based on the Alg17c crystal structure in complex with the alginate trisaccharide (Protein Data Bank 4OJZ) predicted the $\mathrm{p} K_{a}$ values of the corresponding tyrosine and histidine to be 15.4 and 3.84, respectively. Based on these estimations and given that Smlt2602 exhibited optimal activity at pH 8.5 (see Additional file 1: Figure S1 for relative activity against poly-ManA as a function of $\mathrm{pH})$, it is unlikely that $\mathrm{Tyr}^{455}$ would be deprotonated and therefore cannot act as the general base during catalysis. Conversely, His418 would be deprotonated and able to fulfill the role of the catalytic base. Thus, we conclude that the most likely catalytic mechanism for Smlt2602 is as follows: $\mathrm{Asn}^{207}$ and $\mathrm{His}^{208}$ act as neutralizing groups, $\mathrm{His}^{418}$ acts as the general base, and $\mathrm{Tyr}^{264}$ acts as the general acid.

\section{Isolation of an $\mathrm{H} 208 \mathrm{~F}$ mutant that exhibits significant poly- $\beta$-D-glucuronic acid activity}

Mutation of $\mathrm{His}^{208}$ to Phe resulted in an overall reduction in enzymatic activity toward alginate and each of its block structures (Fig. 3a; Table 1). However, the ratio of poly-ManA to poly-GulA activity shifted from 3.5 for WT to 20 for $\mathrm{H} 208 \mathrm{~F}$, indicating that mutation of $\mathrm{His}^{208}$ significantly influenced the substrate specificity of Smlt2602 at the expense of overall enzymatic activity (Fig. 3a; Table 1). The N€2 of the corresponding histidine in $\operatorname{Alg} 17 \mathrm{c}\left(\mathrm{His}^{202}\right)$ lies $2.7 \AA$ from the carboxylic O6A, 3.2 $\AA$ from O5, $3.9 \AA$ from the hydroxyl O2, and $4.4 \AA$ from the glycosidic O4 of the +1 ManA sugar. Furthermore the imidazole ring lies parallel to the +2 GulA ring at a distance of $3.8 \AA$ [13]. Given these multiple points of contact between the substrate and the side chain of His ${ }^{208}$, as well as the shift in substrate specificity with respect to poly-ManA, we postulate that $\mathrm{His}^{208}$ of Smlt2602 plays an important role in regulating substrate specificity and merits further evaluation.

Remarkably, substrate specificity analysis of H208F revealed that the mutant lyase exhibited significant enzymatic activity toward poly-GlcA, a non-alginate-based substrate (Fig. 4; Table 1). To our knowledge, no other characterized PL-17 lyase has been shown to cleave polyGlcA [25]. The specific activity of H208F against polyGlcA (77.2 \pm 1.2 units $/ \mathrm{mg}$ ) is a 28-fold increase over WT poly-GlcA activity $(2.8 \pm 0.4$ units $/ \mathrm{mg})$ and is within an order of magnitude of WT alginate activity (328.3 \pm 27.0$)$ (Fig. 4b; Table 1). Moreover, the H208F poly-GlcA activity is on the same order of magnitude as other poly-GlcA specific lyases $[5,38,39]$. Note that the optimal $\mathrm{pH}$ of activity was slightly shifted from $\mathrm{pH} 8.5$ for alginatebased substrates to $\mathrm{pH} 8$ for poly-GlcA (see Additional file 1: Figure S3 for relative activity of Smlt2602 H208F mutant versus $\mathrm{pH}$ against poly-GlcA). Substrate-dependent variations in optimal $\mathrm{pH}$ have been demonstrated before in PLs and may be indicative of slightly different binding of each substrate [5].

In the interest of determining, if the novel poly-GlcA activity was specific for the phenylalanine mutation or if any mutation of $\mathrm{His}^{208}$ would recapitulate the $\mathrm{H} 208 \mathrm{~F}$ result, we generated two other mutant lyases ( $\mathrm{H} 208 \mathrm{~A}$ and 


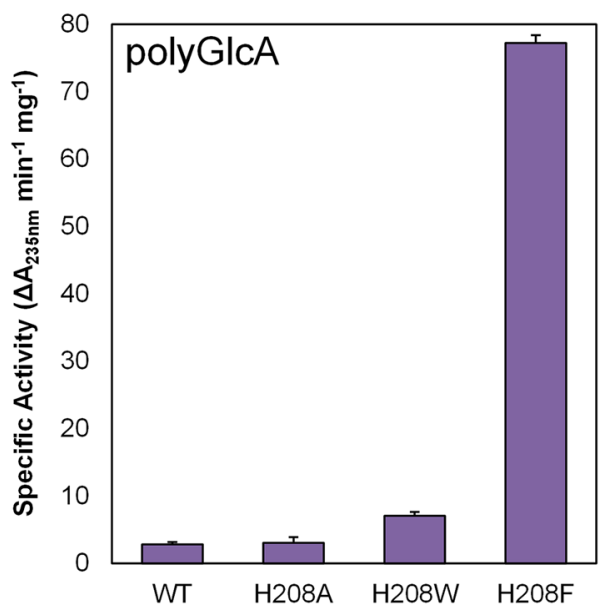

Fig. 4 Enhanced poly-GlcA activity via mutagenesis of $\mathrm{His}^{208}$ residue. Specific activity of WT and H208A, H208W, H208F mutants against $1 \mathrm{mg} / \mathrm{mL}$ poly-GlcA in $20 \mathrm{mM}$ sodium phosphate buffer, pH 8. Enzymatic activity was monitored by change in absorbance at $235 \mathrm{~nm}$. All reactions were performed in triplicate and error is reported as standard deviation

H208W) and measured their ability to digest poly-GlcA. H208A was indistinguishable from WT, whereas H208 W demonstrated a minute twofold increase in activity (7.1 \pm 0.6 units $/ \mathrm{mg}$ ) (Fig. 4). Thus our results indicate that the novel poly-GlcA activity displayed by mutation of $\mathrm{His}^{208}$ is at least partially specific to the phenylalanine substitution (Fig. 4; Table 1).

\section{Size-exclusion chromatography analysis of carbohydrate products formed by Smlt2602 and H208F revealed an exolytic mode of action}

PLs are known to act on polyuronides endolytically, exolytically, or a combination of both. Endolytic lyases bind to the polysaccharide substrate internally, cleave one glycosidic bond, and detach, resulting in the simultaneous accumulation of multiple unsaturated products of various sizes [38, 40-42] (Fig. 5a). Exolytic enzymes bind to the nonreducing end of the polysaccharide substrate and cleave off mono-, di-, or trisaccharides until the entire macromolecule has been digested, resulting in the accumulation of predominantly one unsaturated product, with little to no accumulation of differently sized oligosaccharides [11, 42-44] (Fig. 5b). The hyaluronate lyases of Streptococcus sp. exhibit a hybrid mechanism, in which hyaluronan is digested by an initial random endolytic cleavage and subsequent exolytic processing into disaccharides [31]. Multiple PL-17 lyases, including Alg17c, have been shown to act exolytically on their alginate substrates, reducing the polysaccharide to unsaturated monomers [45-47]. Due to Smlt2602 belonging to the same family and sharing $50 \%$ sequence identity with $\mathrm{Alg} 17 \mathrm{c}$, we predicted that Smlt2602 is an exolytic lyase.

To test this hypothesis, the carbohydrate products formed during Smlt2602 cleavage of both poly-ManA and poly-GlcA were analyzed by size-exclusion chromatography (Fig. 6). Briefly, poly-ManA and poly-GlcA was digested with WT and $\mathrm{H} 208 \mathrm{~F}$, respectively. After $6 \mathrm{~h}$, the reaction mixture was separated on a TSKgel SuperOligoPW column and unsaturated products were detected by monitoring absorbance at $235 \mathrm{~nm}$. A series of molecular weight standards were also run to assist in determining the molecular weight of the sugar products (Fig. 6, dashed vertical lines). The most prominent peak observed for poly-ManA and poly-GlcA eluted between the peaks of D-glucuronic acid and xylose, which have molecular weights of 194 and $150 \mathrm{Da}$, respectively. A calibration curve was generated from the molecular weight standards, and the molecular weight of this most prominent species was estimated to be $166 \mathrm{Da}$, in close agreement with the molecular weight of ManA and GlcA unsaturated monomers (176 Da). This is consistent with the exolytic mode of action observed in other PL-17 lyases.

As stated previously, exolytic cleavage usually results in the accumulation of a single sugar species. A single species was detected for the poly-GlcA run (Fig. 6 bottom), whereas an additional peak of lower abundance was detected for the poly-ManA run. This second species was estimated to have molecular weight of $378 \mathrm{Da}$, in close agreement with the molecular weight of a disaccharide comprised a saturated and unsaturated uronic acid (368 Da) (Fig. 6 top). Given that WT demonstrates the lowest activity toward poly-G (Fig. 1b; Table 1), it is possible this secondary product is a $\Delta$-GulA dimer. Regardless, the accumulation of unsaturated monomers is indicative of an exolytic mode of action for Smlt2602 and $\mathrm{H} 208 \mathrm{~F}$ against poly-ManA and poly-GlcA, respectively. To our knowledge, this is the first characterized bacterial PL with exolytic alginate and poly-GlcA activity [25].

\section{Kinetic parameters of Smlt2602 and H208F}

In order to evaluate the effect, the $\mathrm{His}^{208}$ to Phe mutation had on substrate binding and product conversion, the Michaelis constant $\left(K_{M}\right)$ and the turnover number $\left(k_{\mathrm{CAT}}\right)$ of WT and H208F were determined for alginate, polyManA, poly-GulA, poly-MG, and poly-GlcA (Table 2). The two parameters are dependent on one another and their ratio $\left(k_{\mathrm{CAT}} / K_{M}\right)$ is considered a measure of enzyme efficiency $[30,32]$. Note that although $K_{M}$ may approximate substrate affinity, it is also affected by other rate and equilibrium constants, and therefore $k_{\mathrm{CAT}} / K_{M}$ is used as a descriptive parameter for substrate binding [48]. As can be seen is Table 2, the $k_{\text {CAT }}$ values for WT trend 


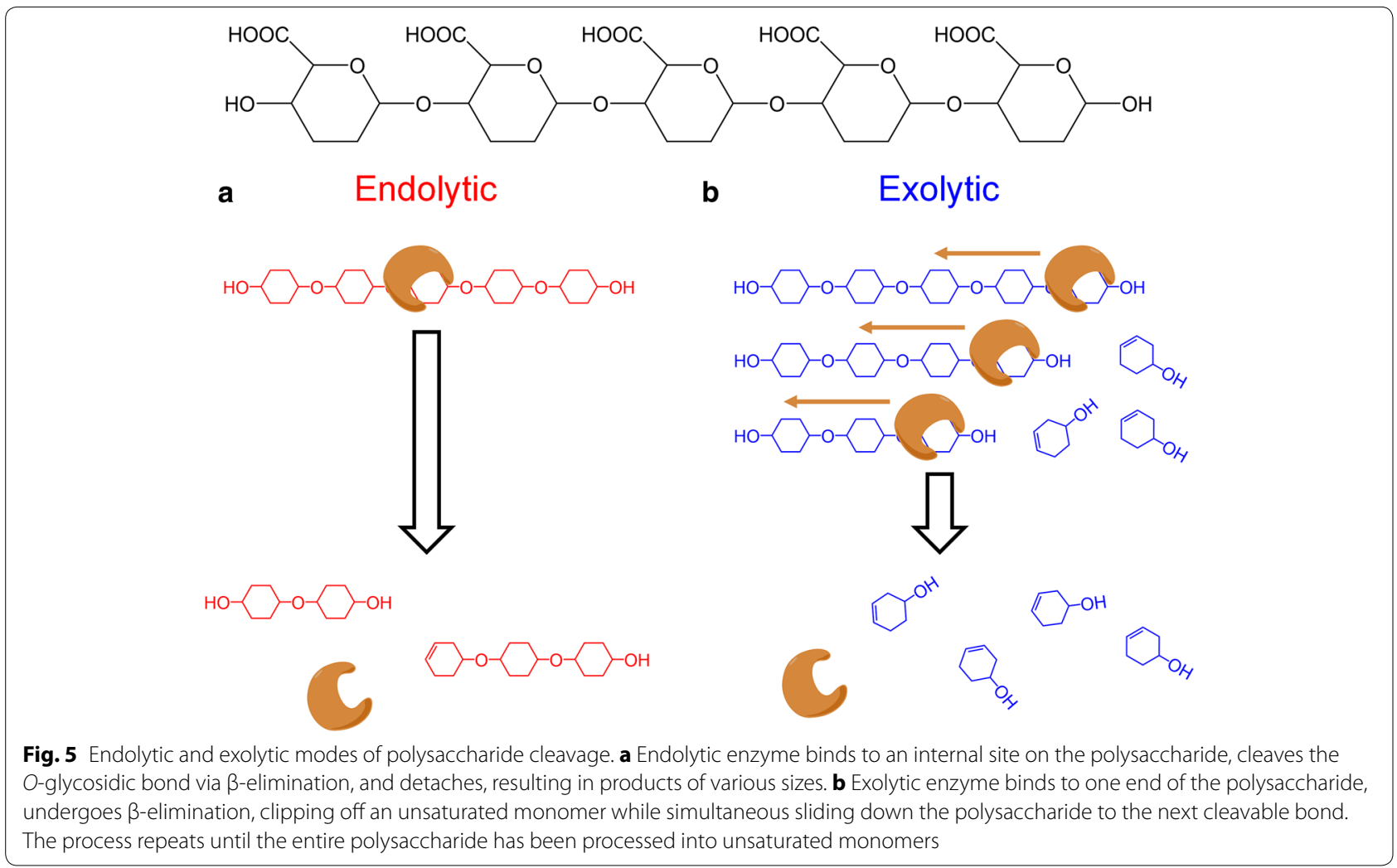

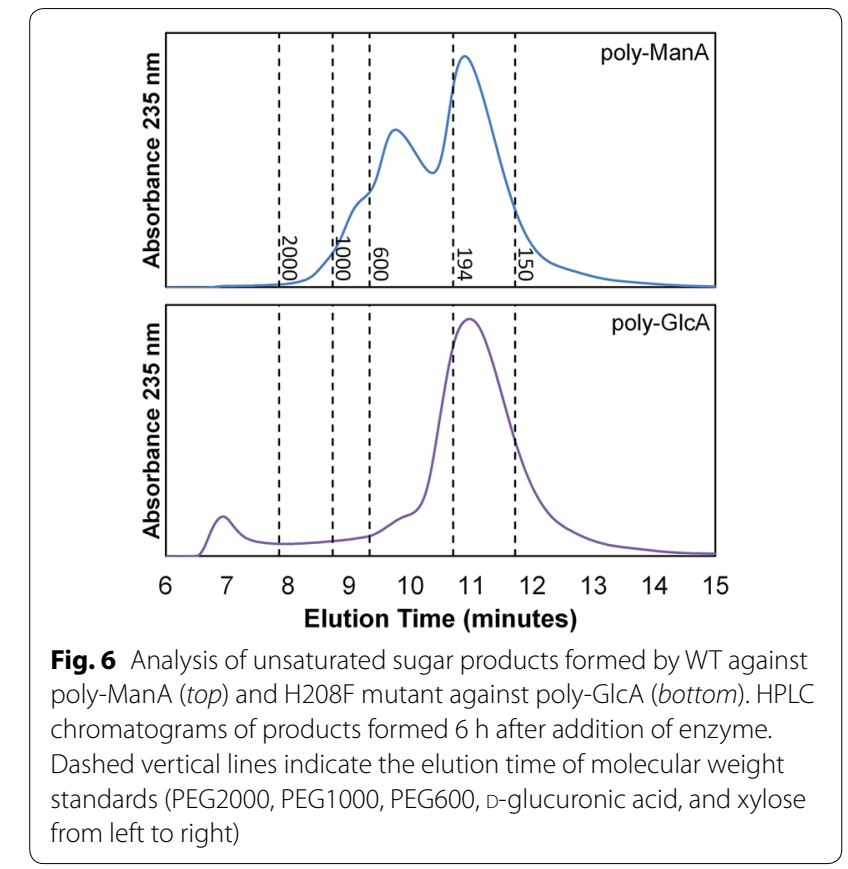

with the specific activity measured for each substrate (Fig. 1b), with poly-ManA having the highest turnover rate $\left(62.2 \mathrm{~s}^{-1}\right)$ and alginate, poly-GulA, and poly-MG all having approximately half that rate $(34.8,34.6$, and $22.2 \mathrm{~s}^{-1}$, respectively). WT also exhibited residual turnover of poly-GlcA $\left(0.4 \mathrm{~s}^{-1}\right)$. The $K_{M}$ values of WT for the alginate-based substrates also followed a similar trend, with poly-ManA having the lowest $K_{M}$ value $(0.41 \mathrm{mM})$, reflective of the highest substrate affinity. Interestingly, the $K_{M}$ value of poly-GulA $(5.99 \mathrm{mM})$ is at least fourfold higher than any other substrate, including poly-GlcA. Thus, the enzyme efficiency of WT toward poly-GulA $(5.8 \mathrm{mM} / \mathrm{s})$ was the lowest for all the alginate-based substrates and is indicative of poor poly-GulA binding. Overall, our results demonstrate that WT is an alginatespecific lyase, with highest substrate affinity and product turnover for poly-ManA (Table 2).

The H208F mutant demonstrated decreases in $k_{\mathrm{CAT}}$ for all alginate-based substrates relative to WT (Table 2), in agreement with the measured specific activities for each substrate (Fig. 3a). Furthermore, H208F displayed a 15-fold increase in turnover rate for poly-GlcA (Table 2), reflective of the 28 -fold increase in specific activity relative to WT (Fig. 4). This increase in $k_{\mathrm{CAT}}$ was coupled with a decrease in $K_{M}$ from $1.4 \mathrm{mM}$ for WT to 0.57 for $\mathrm{H} 208 \mathrm{~F}$, on pair with the binding of poly-ManA $(0.41 \mathrm{mM})$ for each enzyme. The increase in turnover rate and decrease in Michaelis constant resulted in 35-fold increase in enzyme efficiency toward poly-GlcA, from 
Table 2 Substrate specificity ratios of mutant lyase

\begin{tabular}{lrl}
\hline & ManA/GulA & ManA/MG \\
\hline WT & $3.5 \pm 0.1$ & $2.3 \pm 0.1$ \\
R143L & $2.6 \pm 0.1$ & $1.5 \pm 0.1$ \\
Q153A & $15.1 \pm 1.5$ & $6.2 \pm 0.3$ \\
Q153N & $3.9 \pm 0.1$ & $3.0 \pm 0.3$ \\
L155A & $21.1 \pm 0.6$ & $6.7 \pm 0.1$ \\
L155N & $12.1 \pm 0.8$ & $3.9 \pm 0.1$ \\
N201L & $6.6 \pm 0.6$ & $3.6 \pm 0.1$ \\
H206F & $2.6 \pm 0.1$ & $2.0 \pm 0.1$ \\
Y258F & $3.9 \pm 0.2$ & $2.7 \pm 0.2$ \\
Y263F & $15.7 \pm 1.7$ & $6.2 \pm 0.6$ \\
\hline
\end{tabular}

Ratios were calculated by dividing the poly-ManA activity of each mutant over the poly-GulA or poly-MG activity

0.3 for WT to 10.6 for H208F. This value for enzyme efficiency is on the same order of magnitude as WT efficiency toward alginate (Table 2) and within one order of magnitude of the value for the highly active, poly-GlcA specific, and endolytic lyase Smlt1473 [5]. Overall, our results demonstrate that $\mathrm{H} 208 \mathrm{~F}$ is multifunctional $\mathrm{PL}$, with significant binding and exolytic turnover of both alginate- and glucuronan-based substrates (Table 2), which, to our knowledge, is unprecedented for PLs [25].

It is interesting to consider the structural differences between the GlcA and ManA sugar residues in the context of explaining the increase in poly-GlcA activity observed for the H208F mutant. The only structural difference between the ManA and GlcA monomers is the positioning of the $\mathrm{C} 2$ hydroxyl group. In ManA, the hydroxyl group points up, axial to the sugar ring, whereas in GlcA, it points down-equatorial to the ring [8]. As stated previously, the $N \in 2$ of the histidine in Alg17c corresponding to $\mathrm{His}^{208}$ in Smlt2602 lies $3.9 \AA$ away from the C2 hydroxyl group of the +1 ManA sugar and the two atoms likely form a hydrogen bond. This bond would be disrupted upon mutation to phenylalanine and may account for the reduced enzymatic efficiency of the $\mathrm{H} 208 \mathrm{~F}$ mutant toward poly-ManA. Given that ManA and GlcA are C2 epimers, the $N \epsilon 2$ of $\mathrm{His}^{208}$ would be unlikely to form a hydrogen bond with the $\mathrm{C} 2$ hydroxyl of the +1 sugar due to the hydroxyl group pointing in the opposite direction relative to the sugar ring. Therefore, the His ${ }^{208}$ mutation would not affect binding of poly-GlcA at this position. This, of course, does not explain the novel poly-GlcA activity of $\mathrm{H} 208 \mathrm{~F}$, but it is an example of how the same point mutation can have divergent effects of two chemically related substrates. A high-resolution crystal structure of Smlt2602 and H208F in complex with poly-ManA and poly-GlcA would be required to fully elucidate the mechanism behind the enhanced poly-GlcA activity of H208F.

\section{Substrate analysis of additional putative substrate-binding mutants}

Residues located in the active site cleft but not directly involved in the $\beta$-elimination mechanism are thought to participate in substrate binding and alignment with respect to the catalytically active residues to allow for optimal cleavage of the glycosidic bond. Mutation of such residues in the endolytic lyase Smlt1473 resulted in significant changes in substrate specificity and overall enzyme activity and thus could be applied to designing highly active and specific endolyases [12]. In an effort to explore whether the same principle applies for exolytic lyases and given that the H208F mutant exhibited significant poly-GlcA activity that was absent from WT, we mutated seven additional putative substrate-binding residues (Arg ${ }^{143}, \mathrm{Gln}^{153}$, Leu ${ }^{155}, \mathrm{Asn}^{201}, \mathrm{His}^{206}, \mathrm{Tyr}^{258}$, $\mathrm{Tyr}^{263}$, and $\mathrm{His}^{418}$ ), identified via homology modeling (Fig. 2). A total of ten mutants were prepared (R143L, Q153A, Q153N, L155A, L155N, N201L, H206F, Y258F, $\mathrm{Y} 263 \mathrm{~F}$, and $\mathrm{H} 418 \mathrm{~F}$ ) with some residues being mutated to two different amino acids. Although all of the additional mutants, with the exception of N201L against polyManA, displayed an overall decrease in activity that was independent of substrate, a number of them did exhibit significant changes in substrate specificity toward alginate and its three block types (Table 1).

Given that WT is most active against poly-ManA, we analyzed the mutants by calculating the ratio of polyManA activity to poly-GulA activity or poly-MG, designated " $R_{M / G}$ " and " $R_{M / M G}$ " respectively (Table 3 ). All $R_{M / G}$ and $R_{M / M G}$ values were greater than unity, and therefore, all mutant lyases remained most active toward poly-ManA compared to poly-GulA and poly-MG. The $R_{M / G}$ and $R_{M / M G}$ values for $W T$ are 3.5 and 2.3 , respectively. An increase in either value over WT represented a shift in substrate specificity toward poly-ManA over the other substrate. Four of the mutants (R143L, Q153N, $\mathrm{H} 206 \mathrm{~F}$, and $\mathrm{Y} 258 \mathrm{~F}$ ) displayed minimal changes in $\mathrm{R}_{\mathrm{M} / \mathrm{G}}$ and $R_{M / M G}$ relative to $W T$. The rest exhibited significant increases in both $R_{M / G}$ and $R_{M / M G}$, indicating that the point mutations were causing the mutant lyases to favor ManA-ManA linkages, which are the predominant bond found in poly-ManA, over GulA-GulA, ManAGulA, and GulA-ManA linkages found in the other two substrates (Table 3 ). Given that $R_{M / G}$ and $R_{M / M G}$ trended together, we focused our analysis on comparing the $R_{M / G}$ ratio to the overall poly-ManA activity (Fig. 7).

The mutant lyases were found to group into four general categories, colored coded in Fig. 7. The first category comprised R143L, Q153N, H206F, and Y258F (Fig. 7, red). These mutants displayed minute changes in substrate specificity compared to WT and exhibited $\leq 52 \%$ overall poly-ManA activity. Thus, mutation of 
Table 3 Substrate specificity of Smlt2602 mutants

\begin{tabular}{|c|c|c|c|c|c|}
\hline & \multicolumn{5}{|c|}{ Specific activity $\left(\Delta \mathrm{A}_{235 \mathrm{~nm}} \mathrm{~min}^{-1} \mathrm{mg}^{-1}\right)$} \\
\hline & Alginate & Poly-ManA & Poly-GulA & Poly-MG & Poly-GIcA \\
\hline WT & $328.3 \pm 27.0$ & $661.9 \pm 29.1$ & $190.3 \pm 8.3$ & $289.3 \pm 9.8$ & $2.8 \pm 0.4$ \\
\hline \multicolumn{6}{|c|}{ Possible involvement in $\beta$-elimination mechanism } \\
\hline Y455F & $3.6 \pm 0.3$ & $4.0 \pm 0.4$ & $15.3 \pm 1.4$ & $11.5 \pm 0.1$ & $0.2 \pm 0.1$ \\
\hline \multicolumn{6}{|c|}{ Enhanced poly-GlcA activity } \\
\hline H208A & $48.2 \pm 3.5$ & $83.2 \pm 4.2$ & $6.3 \pm 0.8$ & $3.6 \pm 0.4$ & $3.1 \pm 0.8$ \\
\hline $\mathrm{H} 208 \mathrm{~W}$ & $185.3 \pm 11.8$ & $490.8 \pm 4.1$ & $48.5 \pm 1.0$ & $117.1 \pm 7.8$ & $7.1 \pm 0.6$ \\
\hline H208F & $88.4 \pm 4.0$ & $189.7 \pm 4.1$ & $9.5 \pm 0.8$ & $23.3 \pm 1.0$ & $77.2 \pm 1.2$ \\
\hline \multicolumn{6}{|c|}{ Additional putative substrate-binding residues } \\
\hline R143L & $224.1 \pm 13.9$ & $245.4 \pm 15.3$ & $95.5 \pm 2.5$ & $159.0 \pm 9.2$ & $1.1 \pm 0.3$ \\
\hline Q153A & $129.0 \pm 3.5$ & $456.4 \pm 26.4$ & $30.3 \pm 3.5$ & $73.6 \pm 4.4$ & $0.2 \pm 0.1$ \\
\hline Q153 N & $113.8 \pm 9.9$ & $323.7 \pm 17.1$ & $83.4 \pm 2.6$ & $108.7 \pm 10.7$ & $0.9 \pm 0.2$ \\
\hline L155A & $158.5 \pm 9.5$ & $597.6 \pm 46.9$ & $28.4 \pm 0.9$ & $89.6 \pm 1.4$ & $0.3 \pm 0.1$ \\
\hline L155 N & $125.6 \pm 12.6$ & $512.7 \pm 23.2$ & $42.4 \pm 2.9$ & $131.9 \pm 4.8$ & $0.3 \pm 0.2$ \\
\hline N201L & $380.1 \pm 9.2$ & $915.8 \pm 31.9$ & $138.6 \pm 12.9$ & $255.3 \pm 9.9$ & $3.1 \pm 0.7$ \\
\hline $\mathrm{H} 206 \mathrm{~F}$ & $73.9 \pm 2.3$ & $147.8 \pm 3.9$ & $57.0 \pm 2.9$ & $73.2 \pm 0.2$ & $0.4 \pm 0.1$ \\
\hline Y258F & $173.4 \pm 9.1$ & $346.2 \pm 16.1$ & $88.7 \pm 6.0$ & $127.7 \pm 9.5$ & $0.5 \pm 0.1$ \\
\hline Y263F & $36.1 \pm 0.4$ & $83.5 \pm 1.7$ & $5.3 \pm 0.6$ & $13.5 \pm 1.4$ & $0.2 \pm 0.1$ \\
\hline
\end{tabular}

Purified wild-type and mutant Smlt 2602 was added to $1 \mathrm{mg} / \mathrm{mL}$ of each substrate in $20 \mathrm{mM}$ sodium phosphate buffer at pH 8.5 for alginate-based polysaccharides and $\mathrm{pH} 8$ for poly-GlcA. Enzymatic activity was monitored by absorbance at $235 \mathrm{~nm}$. In addition to the substrates listed below, each enzyme was tested against polyGalA, heparin, heparan sulfate, and hyaluronic acid, with no detectable activity. All reactions were performed in triplicate, and error is reported as standard deviation

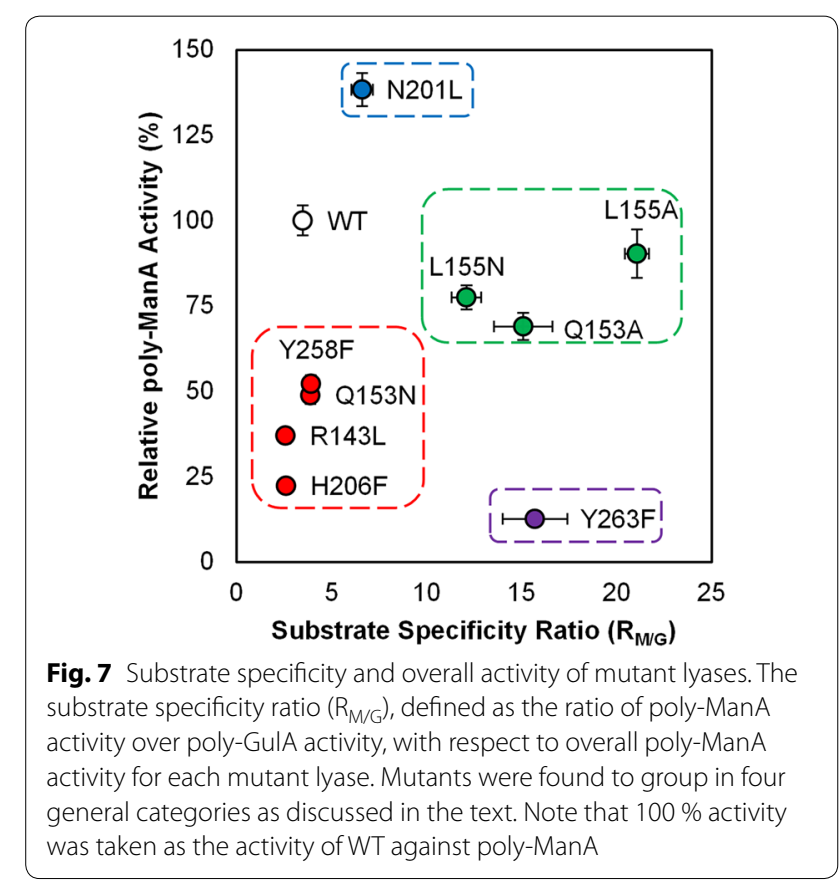

these residues impedes either binding or catalysis, independent of substrate. The second category comprised Q153A, L155A, and L155 N (Fig. 7, green). The overall poly-ManA of each of these mutants was $\geq 68 \%$, and the
$R_{M / G}$ values were $\geq 12$, indicating these lyases were highly selective for poly-ManA with overall activity on pair with WT. The third category comprised Y263F (Fig. 7, purple) which displayed the lowest overall poly-ManA activity (12.6\%) and $\mathrm{R}_{\mathrm{M} / \mathrm{G}}$ value of 15.7 , indicating the mutant is highly selective for poly-ManA, but catalysis is severely impeded. Given its close proximity to the catalytic acid $\left(\mathrm{Tyr}^{264}\right)$, it is likely that $\mathrm{Tyr}^{263}$ plays an indirect role in catalysis (Fig. 2). The fourth category comprised N201L (Fig. 7, blue), the only mutant to exhibit an increase in overall poly-ManA activity (138\%). Furthermore, the $\mathrm{R}_{\mathrm{M} / \mathrm{G}}$ value was 6.6, almost twice that of WT. Therefore, this mutant is both highly active and specific for polyManA. Overall our results show that it is possible to modulate the substrate specificity of Smlt2602 through mutation of residues located near the catalytic core.

\section{Conclusions}

In summary, we describe the characterization of a PL-17 polysaccharide lyase from Stenotrophomonas maltophilia K279a which exolytically degrades alginate. Through homology modeling we identified a key residue $\left(\mathrm{His}^{208}\right)$ thought to be involved in substrate binding and upon mutation, we were able to introduce novel exolytic polyGlcA activity into the lyase. Numerous exolytic polysaccharide lyases have been described which degrade alginate [23], poly-GalA [49], hyaluronan [31], heparan 
sulfate [50], and chondroitin sulfate [51]. To our knowledge, no lyase has even been described that exolytically digests both alginate and poly-GlcA, and as such, Smlt2602 could be utilized to efficiency digest algae polysaccharides into fermentable monomers for biofuel production. Furthermore, our work demonstrates the possibility of engineering substrate specificity into lyases, which has applications in energy and chemical production, but in the design of glycan-based chemicals as well.

\section{Additional file}

Additional file 1. This file contains additional figures $(S 1, S 2, S 3)$ referenced in the manuscript; Figure $\mathbf{S 1}$. Optimal pH of WT against polyManA. Figure S2. Circular dichroism spectrum of wild-type and mutant Smlt2602. Figure S3. Optimal pH of H208F mutant against poly-GlcA.

\section{Abbreviations}

ManA: D-mannuronic acid; GulA: L-guluronic acid; GlCA: D-glucuronic acid; PL: polysaccharide lyase; WT: wild-type; poly-GalA: poly-a-D-galacturonic acid.

\section{Authors' contributions}

LCM, EBW, and BWB designed and interpreted experiments, collected and analyzed data, prepared figures, and wrote the manuscript. All authors read and approved the manuscript.

\section{Author details}

1 Program in Bioengineering, Lehigh University, B320 lacocca Hall, 111 Research Drive, Bethlehem, PA 18015, USA. ${ }^{2}$ Department of Chemical and Biomolecular Engineering, Lehigh University, B320 lacocca Hall, 111 Research Drive, Bethlehem, PA 18015, USA.

\section{Competing interests}

The authors declare that they have no competing interests.

\section{Funding}

This work was supported by the National Science Foundation (CBET 1452855) and through a Faculty Innovation Grant (FIG) from Lehigh University. Additional support for EBW was provided by the Claire Luce Booth Undergraduate Research Scholars program.

Received: 14 December 2015 Accepted: 9 February 2016

Published online: 24 February 2016

\section{References}

1. Medipally SR, Yusoff FM, Banerjee S, Shariff M. Microalgae as sustainable renewable energy feedstock for biofuel production. BioMed Res Int. 2015;2015:519513.

2. Singh A, Olsen SI. A critical review of biochemical conversion, sustainability and life cycle assessment of algal biofuels. Appl Energy. 2011;88(10):3548-55.

3. Wargacki AJ, Leonard E, Win MN, Regitsky DD, Santos CN, Kim PB, Cooper SR, Raisner RM, Herman A, Sivitz AB, et al. An engineered microbial platform for direct biofuel production from brown macroalgae. Science (New York, NY). 2012;335(6066):308-13.

4. Ray B. Polysaccharides from Enteromorpha compressa: isolation, purification and structural features. Carbohydr Polym. 2006;66(3):408-16.

5. MacDonald LC, Berger BW. A polysaccharide lyase from Stenotrophomonas maltophilia with a unique, pH-regulated substrate specificity. J Biol Chem. 2014;289(1):312-25.

6. Lee SI, Choi SH, Lee EY, Kim HS. Molecular cloning, purification, and characterization of a novel polyMG-specific alginate lyase responsible for alginate MG block degradation in Stenotrophomas maltophilia KJ-2. Appl Microbiol Biotechnol. 2012;95(6):1643-53.
7. Ryu M, Lee EY. Saccharification of alginate by using exolytic oligoalginate lyase from marine bacterium Sphingomonas sp. MJ-3. J Ind Eng Chem. 2011;17(5-6):853-8.

8. Garron ML, Cygler M. Structural and mechanistic classification of uronic acid-containing polysaccharide lyases. Glycobiology. 2010;20(12):1547-73.

9. Preiss J, Ashwell G. Alginic acid metabolism in bacteria. I. enzymatic formation of unsaturated oligosaccharides and 4-deoxy-L-erythro-5-hexoseulose uronic acid. J Biol Chem. 1962;237:309-16.

10. Tondervik A, Klinkenberg G, Aarstad OA, Drablos F, Ertesvag H, Ellingsen TE, Skjak-Braek G, Valla S, Sletta H. Isolation of mutant alginate lyases with cleavage specificity for di-guluronic acid linkages. J Biol Chem. 2010;285(46):35284-92.

11. Farrell EK, Tipton PA. Functional characterization of AlgL, an alginate lyase from Pseudomonas aeruginosa. Biochemistry. 2012;51(51):10259-66.

12. MacDonald LC, Berger BW. Insight into the role of substrate-binding residues in conferring substrate specificity for the multifunctional polysaccharide lyase Smlt1473. J Biol Chem. 2014;289(26):18022-32.

13. Park D, Jagtap S, Nair SK. Structure of a PL17 family alginate lyase demonstrates functional similarities among exotype depolymerases. J Biol Chem. 2014;289(12):8645-55.

14. Arnold K, Bordoli L, Kopp J, Schwede T. The SWISS-MODEL workspace: a web-based environment for protein structure homology modelling. Bioinformatics. 2006;22(2):195-201.

15. Bordoli L, Kiefer F, Arnold K, Benkert P, Battey J, Schwede T. Protein structure homology modeling using SWISS-MODEL workspace. Nat Protocols. 2008;4(1):1-13.

16. Kiefer F, Arnold K, Kunzli M, Bordoli L, Schwede T. The SWISS-MODEL repository and associated resources. Nucleic Acids Res. 2009; 37(Database issue):D387-92.

17. Schrodinger LLC. The PyMOL molecular graphics system, Version 1.3r1. In.; 2010.

18. Sambrook J, Russell DWDW, Laboratory CSH. Molecular cloning: a laboratory manual. 3rd. ed. Cold Spring Harbor Laboratory; 2001.

19. Wilkins MR, Gasteiger E, Bairoch A, Sanchez JC, Williams KL, Appel RD, Hochstrasser DF. Protein identification and analysis tools in the ExPASy server. Methods Mol Biol. 1999;112:531-52.

20. Fylstra D, Lasdon L, Watson J, Waren A. Design and use of the microsoft excel solver. Interfaces. 1998;28(5):29-55.

21. Weissbach A, Hurwitz J. The formation of 2-keto-3-deoxyheptonic acid in extracts of Escherichia coli B. I. identification. J Biol Chem. 1959;234(4):705-9.

22. Inoue A, Takadono K, Nishiyama R, Tajima K, Kobayashi T, Ojima T. Characterization of an alginate lyase, FIAlyA, from Flavobacterium sp. strain UMI-01 and its expression in Escherichia coli. Marine Drugs. 2014;12(8):4693-712.

23. Jagtap SS, Hehemann JH, Polz MF, Lee JK, Zhao H. Comparative biochemical characterization of three exolytic oligoalginate lyases from Vibrio splendidus reveals complementary substrate scope, temperature, and pH adaptations. Appl Environ Microbiol. 2014;80(14):4207-14.

24. Wong TY, Preston LA, Schiller NL. ALGINATE LYASE: review of major sources and enzyme characteristics, structure-function analysis, biological roles, and applications. Annu Rev Microbiol. 2000;54:289-340.

25. Lombard V, Golaconda Ramulu H, Drula E, Coutinho PM, Henrissat B. The carbohydrate-active enzymes database (CAZy) in 2013. Nucleic acids research. 2014; 42(Database issue):D490-95.

26. Louis-Jeune C, Andrade-Navarro MA, Perez-Iratxeta C. Prediction of protein secondary structure from circular dichroism using theoretically derived spectra. Proteins. 2012;80(2):374-81.

27. Gacesa P. Alginate-modifying enzymes: a proposed unified mechanism of action for the lyases and epimerases. FEBS Lett. 1987;212(2):199-202.

28. Asensio JL, Arda A, Canada FJ, Jimenez-Barbero J. Carbohydrate-aromatic interactions. Acc Chem Res. 2013;46(4):946-54.

29. Shaya D, Zhao W, Garron ML, Xiao Z, Cui Q, Zhang Z, Sulea T, Linhardt RJ, Cygler M. Catalytic mechanism of heparinase II investigated by sitedirected mutagenesis and the crystal structure with its substrate. J Biol Chem. 2010;285(26):20051-61.

30. Nukui M, Taylor KB, McPherson DT, Shigenaga MK, Jedrzejas MJ. The function of hydrophobic residues in the catalytic cleft of Streptococcus pneumoniae hyaluronate lyase. Kinetic characterization of mutant enzyme forms. J Biol Chem. 2003;278(5):3079-88. 
31. Jedrzejas MJ, Mello LV, de Groot BL, Li S. Mechanism of hyaluronan degradation by Streptococcus pneumoniae hyaluronate lyase. Structures of complexes with the substrate. J Biol Chem. 2002;277(31):28287-97.

32. Mikami B, Ban M, Suzuki S, Yoon HJ, Miyake O, Yamasaki M, Ogura K, Maruyama Y, Hashimoto W, Murata K. Induced-fit motion of a lid loop involved in catalysis in alginate lyase A1-III. Acta Crystallogr D Biol Crystallogr. 2012;68(Pt 9):1207-16.

33. Li H, Robertson AD, Jensen JH. Very fast empirical prediction and rationalization of protein pKa values. Proteins. 2005;61(4):704-21.

34. Bas DC, Rogers DM, Jensen $\mathrm{JH}$. Very fast prediction and rationalization of pKa values for protein-ligand complexes. Proteins. 2008;73(3):765-83.

35. Olsson MHM, Sondergard CR, Rostkowski M, Jensen JH. PROPKA3: consistent treatment of internal and surface residues in empirical pka predictions. J Chem Theory Comput. 2011;7(2):525-37.

36. Sondergard CR, Olsson MHM, Rostkowski M, Jensen JH. Improved treatment of ligands and coupling effects in empirical calculation and rationalization of pka values. J Chem Theory Comput. 2011;7(7):2284-95.

37. Harris TK, Turner GJ. Structural basis of perturbed pKa values of catalytic groups in enzyme active sites. IUBMB Life. 2002;53(2):85-98

38. Konno N, Igarashi K, Habu N, Samejima M, Isogai A. Cloning of the Trichoderma reesei cDNA encoding a glucuronan lyase belonging to a novel polysaccharide lyase family. Appl Environ Microbiol. 2009;75(1):101-7.

39. Da Costa A, Michaud P, Petit E, Heyraud A, Colin-Morel P, Courtois B, Courtois J. Purification and properties of a glucuronan lyase from Sinorhizobium meliloti M5N1CS (NCIMB 40472). Appl Environ Microbiol. 2001;67(11):5197-203.

40. Da Costa A, Michaud P, Petit E, Heyraud A, Colin-Morel P, Courtois B, Courtois J. Purification and properties of a glucuronan lyase from Sinorhizobium meliloti M5N1CS (NCIMB 40472). Appl Environ Microbiol. 2001;67(11):5197-203

41. Yoon HJ, Hashimoto W, Miyake O, Okamoto M, Mikami B, Murata K Overexpression in Escherichia coli, purification, and characterization of Sphingomonas sp. A1 alginate lyases. Protein Expr Purif. 2000;19(1):84-90.
42. Preston JF 3rd, Rice JD, Ingram LO, Keen NT. Differential depolymerization mechanisms of pectate lyases secreted by Erwinia chrysanthemi EC16. J Bacteriol. 1992;174(6):2039-42.

43. Miyake O, Hashimoto W, Murata K. An exotype alginate lyase in Sphingomonas sp. A1: overexpression in Escherichia coli, purification, and characterization of alginate lyase IV (A1-IV). Protein Expr Purif. 2003;29(1):33-41.

44. Konno N, Habu N, lihashi N, Isogai A. Purification and characterization of exo-type cellouronate lyase. Cellulose. 2008;15:453-63.

45. Kim HT, Chung JH, Wang D, Lee J, Woo HC, Choi IG, Kim KH. Depolymerization of alginate into a monomeric sugar acid using Alg17C, an exooligoalginate lyase cloned from Saccharophagus degradans 2-40. Appl Microbiol Biotechnol. 2012;93(5):2233-9.

46. Kraiwattanapong J, Ooi T, Kinoshita S. Cloning and sequence analysis of the gene (alyll) coding for an alginate lyase of Pseudomonas sp. OS-ALG-9. Biosci Biotechnol Biochem. 1997;61(11):1853-7.

47. Park HH, Kam N, Lee EY, Kim HS. Cloning and characterization of a novel oligoalginate lyase from a newly isolated bacterium Sphingomonas sp. MJ-3. Marine Biotechnol. 2012;14(2):189-202.

48. Kelly SJ, Taylor KB, Li S, Jedrzejas MJ. Kinetic properties of Streptococcus pneumoniae hyaluronate lyase. Glycobiology. 2001;11(4):297-304.

49. Preston JF, Rice JD, Ingram LO, Keen NT. Differential depolymerization mechanisms of pectate lyases secreted by Erwinia chrysanthemi EC16. J Bacteriol. 1992;174(6):2039-42.

50. Ernst S, Rhomberg AJ, Biemann K, Sasisekharan R. Direct evidence for a predominantly exolytic processive mechanism for depolymerization of heparin-like glycosaminoglycans by heparinase I. Proc Natl Acad Sci USA. 1998;95(8):4182-7.

51. Hamai A, Hashimoto N, Mochizuki H, Kato F, Makiguchi Y, Horie K, Suzuki S. Two distinct chondroitin sulfate ABC lyases. An endoeliminase yielding tetrasaccharides and an exoeliminase preferentially acting on oligosaccharides. J Biol Chem. 1997;272(14):9123-30.

52. Rye CS, Matte A, Cygler M, Withers SG. An atypical approach identifies TYR234 as the key base catalyst in chondroitin AC lyase. Chembiochem. 2006;7(4):631-7.

\section{Submit your next manuscript to BioMed Central and we will help you at every step:}

- We accept pre-submission inquiries

- Our selector tool helps you to find the most relevant journal

- We provide round the clock customer support

- Convenient online submission

- Thorough peer review

- Inclusion in PubMed and all major indexing services

- Maximum visibility for your research

Submit your manuscript at www.biomedcentral.com/submit

\section{() Biomed Central}

